\title{
Time course of altered sensitivity to inhibitory and excitatory agonist responses in the longitudinal muscle-myenteric plexus and analgesia in the guinea pig after chronic morphine treatment
}

\author{
Dane M. Barrett ${ }^{\dagger}$, Hercules T. Maguma $^{\dagger}$ and David A. Taylor* \\ The Department of Pharmacology and Toxicology, The Brody School of Medicine at East Carolina University, Greenville, NC, USA
}

\section{Edited by:}

Trinity Jude Bivalacqua, Johns

Hopkins Hospital, USA

\section{Reviewed by:}

Vladimir Zagorodnyuk, Flinders

University, Australia

Katsuya Hirano, Kyushu University,

Japan

\section{*Correspondence:}

David A. Taylor, Department of Pharmacology and Toxicology, The Brody School of Medicine at East Carolina University, 600 Moye Blvd., Greenville, NC 27834, USA.

e-mail: taylorda@ecu.edu

\section{${ }^{\dagger}$ Present address:}

Dane M. Barrett, Department of Otolaryngology-Head and Neck

Surgery, University of Virginia Medical Center, PO Box 800713,

Charlottesville, VA 22908-0713, USA; Hercules T. Maguma, Department of Pharmacology and Toxicology, Virginia Commonwealth University School of Medicine, 410 North 12th Street,

Room 746, P.O. Box 980613,

Richmond, VA 23298-0613, USA.

\begin{abstract}
Tolerance that develops after chronic morphine exposure has been proposed to be an adaptive response that develops and decays over a defined time course. The present study examined the development of tolerance to the acute hypothermic and analgesic effects of morphine and correlated the time course for the desensitization in vivo with the reduced responsiveness to DAMGO and 2-CADO and increased responsiveness to nicotine of the longitudinal muscle/myenteric plexus (LM/MP) preparation in vitro. Assessment was performed at various times after morphine or placebo pellet implantation. Morphine produced a modest hypothermic response to which no tolerance developed. However, the development of tolerance to the analgesic effect of morphine, the inhibitory effect of DAMGO and CADO on neurogenic twitches of the LM/MP and hypersensitivity to the contractile response to nicotine was observed to occur in a time-dependent manner. The alterations in sensitivity to DAMGO, nicotine, and responsiveness to morphine analgesia occurred between days 4 and 10 and returned to normal by day 14 post-implantation. In contrast, sensitivity of LM/MP preparations to 2-CADO displayed a similar time-dependent onset but the tolerance persisted beyond 14 days after implantation. These data suggest that the heterologous tolerance that develops after chronic morphine treatment is time-dependent and persistent but, ultimately returns to normal in the absence of any intervention. Furthermore, the data suggest that the basis of the adaptive phenomenon may involve multiple cellular mechanisms including the modulation of cell excitability and normal physiology but the consequences of the adaptation extend to all effects of the agonist.
\end{abstract}

Keywords: morphine tolerance, longitudinal muscle/myenteric plexus, analgesic tolerance, guinea pig, hypothermia, adaptation, opioid receptors, nicotinic receptors

\section{INTRODUCTION}

The use of opioids is associated with the development of tolerance, the extent which varies depending on the pharmacological effect. In humans, tolerance has been observed to various pharmacological effects of morphine like analgesia and euphoria whereas tolerance to miosis, respiration depression, and its constipating effect does not seem to be evident. Cellular mechanisms associated with the development of tolerance to opioids in animal models and humans include but not limited to kinase-related (PKA, PKC, and G protein-coupled receptor kinase) phosphorylation of the receptor and other cellular proteins, beta-arrestin-facilitated downregulation of the receptor, alteration of endogenous ligand levels, changes in resting membrane potential, and cAMP superactivation (Connor et al., 2004). However, the extent to which each or all of these potential mechanisms contributes to the final phenotype and whether the type of tolerance observed in one tissue or model system is also observed in all the systems where the agonist exerts an action has not been adequately explored. Though cellular adaptation observed in the development of tolerance does not appear to be similar in different cells/tissues/systems, it has been observed that the time course for the appearance and disappearance of the altered sensitivity may be comparable for some pharmacological effects (Li et al., 2010).

Opioid tolerance in the guinea longitudinal muscle/myenteric plexus (LM/MP) is associated with the development of nonspecific supersensitivity to excitatory agonists (Schulz and Goldstein, 1973; Johnson et al., 1978) and non-specific subsensitivity to inhibitory agents (Taylor et al., 1988; Leedham et al., 1992) that has been related to a partial membrane depolarization of the myenteric "S" neurons (Li et al., 2010). In addition, it appears that there may be an association between the time course over which the change in responsiveness and the reduction in the abundance of the alpha 3 subunit of the $\mathrm{Na}^{+} / \mathrm{K}^{+}$ATPase occurs in the LM/MP model (Li et al., 2010). However, no studies have determined whether the time course for the development of tolerance in vivo correlates with the time course observed for the loss of responsiveness and development of enhanced responsiveness to nicotine of the LM/MP in vitro. Indeed, the studies that investigated the enhanced responsiveness to excitatory substances only evaluated the responsiveness 7 days after morphine pellet implantation but did not explore any 
other time periods (Schulz and Goldstein, 1973; Johnson et al., 1978).

We previously demonstrated, in longitudinal studies over a span of 21 days, that chronic in vivo treatment with morphine using different treatment paradigms results in the development of quantitatively and qualitatively similar heterologous tolerance in the guinea LM/MP preparation that was first observed between 4 and 7 days, and disappeared by day 14 (Li et al., 2010). We have also previously shown that chronic parenteral treatment with morphine leads to the development of tolerance to the analgesic effects of acute morphine (Maguma and Taylor, 2011) but did not determine whether similar tolerance developed after pellet implantation or explore the time course over which the change occurred. The goal of the present study was to test the hypothesis that chronic in vivo exposure to opioids through pellet implantation would produce a qualitatively similar progression in the appearance, maintenance, and disappearance of enhanced sensitivity to excitatory and reduced sensitivity to inhibitory agents in the LM/MP, and to the analgesic and hypothermic effect of morphine in vivo. A corollary hypothesis to be tested was that the time course over which tolerance developed in these in vivo and in vitro models would be similar and point to a non-specific mechanism underlying the development of opioid tolerance that would be consistent with a general change in cellular excitability across different tissues expressing the mu-opioid receptor. To this end, we have investigated and compared the time course for the alteration in sensitivity to inhibitory (DAMGO and 2-chloroadenosine [2-CADO]) and the excitatory agent nicotine in the longitudinal musclemyenteric plexus (LM/MP) preparation and the changes in the response of the animal to the analgesic and hypothermic effects of morphine.

\section{MATERIALS AND METHODS SUBJECTS}

All experimental procedures employing animals were reviewed and approved by the Institutional Animal Care and Use Committee of the Brody School of Medicine at East Carolina University and were conducted in accordance with the guidelines for the humane use of animals in research [NIH "Public Health Service Policy on Humane Care and Use of Laboratory Animals" (revised 2002)]. Dunkin-Hartley guinea pigs (Charles-River labs; Raleigh, NC, USA) of either sex weighing 200-450 g were used in the study. The animals were housed two per cage with access to food and water ad libitum. The guinea pigs were kept in the animal facility for 1 week to permit acclimation prior to initiation of the treatment. Every effort was made to reduce the use of animals to the minimum number required to achieve sufficient statistical power.

\section{PROCEDURES}

\section{Morphine pellet implantation}

Guinea pigs were anesthetized with isoflurane, administered by inhalation, and one morphine- or lactose-containing pellet per $100 \mathrm{~g}$ of body weight was implanted subcutaneously in the flank. The animals were allowed to recover then returned to the animal facility until the day of the experiment.

\section{Longitudinal smooth musc/e/myenteric plexus preparations}

The LM/MP from treated animals was removed and isolated as previously (Taylor et al., 1988). Segments of the ileum were obtained from animals sacrificed by decapitation following isoflurane anesthesia. The abdomen was opened to expose the cecum. The $10 \mathrm{~cm}$ section of ileum closest to the cecum was removed and discarded, and $2-4 \mathrm{~cm}$ segments of ilea from the adjacent $10 \mathrm{~cm}$ of ileum were used for the LM/MP preparations. The segments of ilea were threaded onto a glass rod and, using a cotton swab moistened with Krebs-Henseleit solution, the LM/MP was carefully stripped tangentially from the point of mesenteric attachment until the muscle-nerve preparation was detached from the total area of the ileum.

The resulting sheet of LM/MP was tied at each end with a fine thread, passed through platinum-ring electrodes, and placed in a 10 -ml organ bath containing Krebs buffer solution. One thread was tied to a Grass FT.03 force transducer and the other fixed to a tissue holder. The output of the force transducer was delivered to a computer and digitized using the PowerLab/Chart 5 computer program (AD Instruments, Colorado Springs, CO, USA) through a four channel power lab system using a four channel Quad bridge converter interface (AD instruments, Colorado Springs, CO, USA).

The tissues were maintained at $37^{\circ} \mathrm{C}$ in a physiological Krebs buffer solution bubbled continuously with a mixture of $95 \%$ $\mathrm{O}_{2} / 5 \% \mathrm{CO}_{2}$ consisting of the following (in $\mathrm{mM}$ ): $\mathrm{NaCl}(117), \mathrm{KCl}$ (4.7), $\mathrm{CaCl}_{2}$ (2.5), $\mathrm{KH}_{2} \mathrm{PO}_{4}$ (1.2), $\mathrm{MgSO}_{4}$ (1.2), $\mathrm{NaHCO}_{3}$ (25), and Dextrose (11.5). A basal tension of $1.0 \mathrm{~g}$ was set for each tissue and isometric tension generated by the muscle was recorded and stored using the PowerLab/Chart 5 program. Neurogenic contractions were elicited via electrical stimulation using supramaximal voltage delivered to the tissue through platinum-ring electrodes using a stimulation system consisting of a Grass S48 stimulator connected to the electrodes by a Med Lab Attenuator and Stimu-splitter. To ensure only nerve endings were stimulated, the following parameters were used: voltage $(50 \mathrm{~V})$; impulse duration $(<1 \mathrm{~ms})$; delay setting (zero); and frequency $(0.1 \mathrm{~Hz})$.

During the initial $1 \mathrm{~h}$ equilibration period, the buffer solution was replaced at $15 \mathrm{~min}$ intervals. Following equilibration, the tissues were exposed to cumulatively increasing concentrations of the inhibitory drugs (final concentrations in the organ bath ranging between $1 \mathrm{nM}$ and $10 \mu \mathrm{M}$ ). Three $5 \mathrm{~min}$ washes followed by three 15 min washes with drug free Krebs-Henseleit solution were performed between concentration-response curves of different drugs permitting full recovery of the amplitude of neurogenic contractions. In each experiment, two LM/MP preparations from each test group of animals were studied simultaneously and the responses of the tissues from the same animal averaged. The effect of each agonist on the amplitude of the neurogenic contractions was calculated as percent inhibition from the original amplitude. Each of the agonists was alternated in sequence to reduce the impact of the sequence of administration upon the calculated $\mathrm{IC}_{50}$ value. Geometric mean $\mathrm{IC}_{50}$ values were calculated and compared among treatment groups as previously described (Taylor et al., 1988).

The procedure outlined above was also used for experiments using nicotine with the only difference being the absence of 
electrical stimulation since nicotine elicited contractions by stimulating ganglionic release of acetylcholine. Nicotine (final bath concentrations ranging between 0.3 and $100 \mu \mathrm{M}$ ) was added in a non-cumulative manner with at least three 5-min washes performed before the next drug concentration was added. Responses were calculated in grams of tension or percent of the maximum contraction for that tissue. The values were used to determine the $\mathrm{EC}_{50}$ (i.e., concentration required to produce $50 \%$ of the maximum response) and to calculate the maximum tension produced by nicotine. Computer assisted analysis of each concentration-response curve using GraphPad Prism ${ }^{\circledR}$ software (GraphPad Software, Inc.) was employed to determine the $\mathrm{IC}_{50}$ or $\mathrm{EC}_{50}$.

\section{Paw pressure mechanical analgesia assessment}

Behavior assessment was performed between 9 and 10 AM on the day of the experiment. Each animal was acclimated to the observation room for $1 \mathrm{~h}$ prior to the initiation of any assessment. The Randall and Selitto (1957) test was used to assess mechanical nociception (Randall and Selitto, 1957). In this test, pressure was manually applied to the plantar surface of the hind paw using a cone-shaped pusher with a rounded tip. The force $(g)$ at which the guinea pig withdrew its hind paw was defined as the paw pressure threshold. A cut-off was set at $600 \mathrm{~g}$ of pressure to prevent tissue damage. Baseline analgesia was determined prior to injecting the challenge drug. Subsequent mean response thresholds were determined at the following time intervals after acute drug administration: $15,30,45,60,90$, and $120 \mathrm{~min}$. Tolerance was defined as a reduction in the antinociceptive effect (decreased threshold for withdrawal) of the challenge dose of agonist such that the maximum amount of pressure required to elicit a paw withdrawal was decreased. The challenge dose employed (morphine, $10 \mathrm{mg} / \mathrm{kg}$ s.c.) was selected based on preliminary studies done to assess the optimum dose required to produce an adequate and quantifiable analgesic response. The magnitude of the tolerance that developed was assessed by calculating the area under the curve (AUC) for the time versus latency response using GraphPad Prism 5. There was no apparent difference in locomotor activity displayed by the subjects in the treatment group in response to the acute drug administration.

\section{Hypothermia assessment}

Assessment of body temperature using a rectal thermometer was performed in a quiet room at an ambient temperature of $25^{\circ} \mathrm{C}$. After a 1-h acclimatization period in the test room, body temperature was measured using a digital rectal thermometer inserted to a constant depth of $2.5 \mathrm{~cm}$. Following thermometer insertion, a 15-s equilibration period was allowed to lapse before the temperature was recorded. Baseline rectal temperature was measured twice prior to drug challenge (morphine $10 \mathrm{mg} / \mathrm{kg}$ s.c.) and subsequently at $15,30,45,60,90$, and 120 min after drug administration. The results were expressed as the mean \pm SEM of the change in temperature $\left({ }^{\circ} \mathrm{C}\right)$ from baseline. The magnitude of the response that developed was assessed by calculating the AUC for the time versus latency response using GraphPad Prism 5. Tolerance was defined as a reduction in the maximum hypothermic effect of the challenge dose of agonist.

\section{DRUGS AND CHEMICALS}

Morphine and placebo pellets were obtained from Dr. K. H. Davis (Research Triangle Institute, Research Triangle Park, NC, USA) through the National Institute on Drug Abuse (NIDA) investigator supply program. Each morphine pellet contained $75 \mathrm{mg}$ morphine whereas a similar amount of lactose replaced morphine in the placebo pellets. Morphine powder (morphine sulfate pentahydrate salt), CADO (2-chloroadenosine), DAMGO ([D-Ala ${ }^{2}$, $\mathrm{N}$-Me-Phe ${ }^{4}, \mathrm{Gly}^{5}$-ol]-Enkephalin acetate), and nicotine (Nicotine hydrogen tartrate) were procured from Sigma-Aldrich Co. (St. Louis, MO, USA). For organ bath studies, solutions of DAMGO, $\mathrm{CADO}$, and nicotine were made by dissolving their respective salts in distilled water. Parenterally administered morphine was dissolved in normal saline.

\section{DATA ANALYSIS}

For the LM/MP organ bath experiments, the sensitivity of a group of tissues to an inhibitory agonist was determined by calculating the mean negative log of the concentration producing 50\% inhibition of the electrically induced contraction $\left(\mathrm{IC}_{50} \pm \mathrm{SEM}\right)$. Percent inhibition was calculated using the mean contraction height at the maximum inhibition following addition of a given concentration of agonist, divided by the average contraction height $1 \mathrm{~min}$ before exposure to the initial dose of that agonist. Differences in sensitivity to a given agonist between two groups of tissues were determined by comparing the geometric mean $\mathrm{IC}_{50}$ values and the mean ratio of geometric mean $\mathrm{IC}_{50}$ values (calculated as the mean antilog of the difference in $\mathrm{IC}_{50}$ values between the two groups). For nicotine stimulation experiments, the $\mathrm{EC}_{50}$ (i.e., the concentration of drug required to produce a contraction magnitude equal to $50 \%$ of the maximum contraction obtained in that tissue) and the maximal isometric tension developed were determined and compared. Significant differences between the test and control groups were determined using unpaired Student's " $t$ " test. Comparison of the magnitude of tolerance produced in each model system was assessed by comparing the ratio of the measurements obtained for the control:morphine treated group with a probability level of $\leq 0.05$ accepted as significantly different.

Statistical analysis of drug-response data (in vivo and in vitro) was performed using a two way ANOVA followed by followed by Bonferroni post hoc test. The Student's $t$-test (unpaired) was used to compare the mean mechanical analgesia threshold between the control and test groups and to compare the effects of the agonists on body temperature. Tolerance was assessed by comparing the maximal analgesic or hypothermic effect observed following acute drug challenge. To assess the magnitude of tolerance, the AUC for the control and the test groups was computed and the control:morphine group AUC ratio was defined as the magnitude of tolerance. A $p$ value $\leq 0.05$ was considered to be statistically significantly different.

\section{RESULTS \\ ASSESSMENT OF THE MORPHINE-INDUCED HYPOTHERMIA FOLLOWING CHRONIC DRUG TREATMENT}

The acute hypothermic effect of morphine $(10 \mathrm{mg} / \mathrm{kg}$ body weight s.c.) was determined by rectal body temperature assessment in the same placebo and morphine pellet-implanted guinea pigs that 
were subjected to mechanical analgesia testing at various times following implantation. Acute challenge with morphine resulted in progressive hypothermia at an ambient temperature of $25^{\circ} \mathrm{C}$ that reached a maximum response between 60 and 90 min after administration. The hypothermic effect of morphine was modest in magnitude and was not affected by the treatment (maximum change in temperature for placebo group $=-1.12 \pm 0.16^{\circ} \mathrm{C}$ versus $-1.32 \pm 0.16^{\circ} \mathrm{C}$ for morphine group). Chronic exposure to morphine did not produce tolerance at any time period depicted (Figures 1A-D). Data obtained in animals at 7, 17, and 21 days post-implantation also failed to demonstrate the development of tolerance (data not shown). However, a small but statistically significant increase in mean body temperature was observed in animals that had been implanted for both 4 days (mean body temperature $=39.4 \pm 0.2^{\circ} \mathrm{C}$ in placebo versus $40.1 \pm 0.1^{\circ} \mathrm{C}$ in morphine animals) and 7 days (mean body temperature $=39.6 \pm 0.1^{\circ} \mathrm{C}$ in placebo versus $39.9 \pm 0.1^{\circ} \mathrm{C}$ in morphine animals, $\left.p \leq 0.05\right)$. Daily assessment confirmed that the change in body temperature was not due to infection or obvious inflammation resulting from the implantation procedure. As indicated in Table 1 and Figure 1E, the ratio of AUC values for the hypothermic effect of morphine between placebo and morphine treated animals was generally close to unity and not statistically significantly different at any time period tested. Though morphine produced a modest hypothermia that was accompanied by wide variability, the data indicate that no tolerance developed to that effect in animals chronically implanted with morphine pellets.

\section{ASSESSMENT OF THE MECHANICAL ANALGESIC RESPONSE FOLLOWING CHRONIC DRUG TREATMENT}

The analgesic effect of morphine $(10 \mathrm{mg} / \mathrm{kg}$ body weight s.c.) to a mechanical stimulus was determined using the paw pressure method (Randall and Selitto, 1957). The analgesic effect of morphine was evident within 15-30 min of drug administration and persisted throughout the observation period $(120 \mathrm{~min})$ with the highest values of pressure required to elicit paw retraction ranging from 400 to $600 \mathrm{~g}$ (Figures 2A-D). The maximum analgesic effect was observed between 30 and $90 \mathrm{~min}$ for both the placebo
A

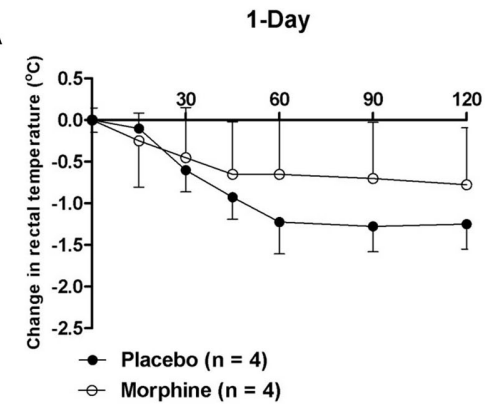

C

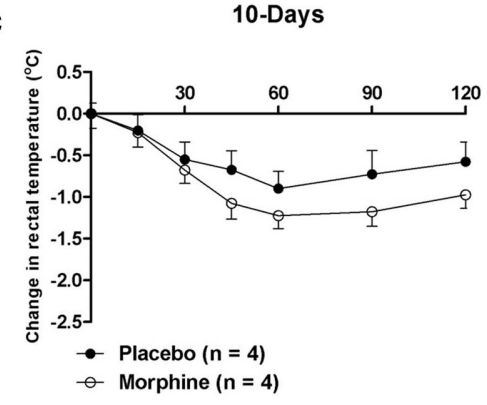

B

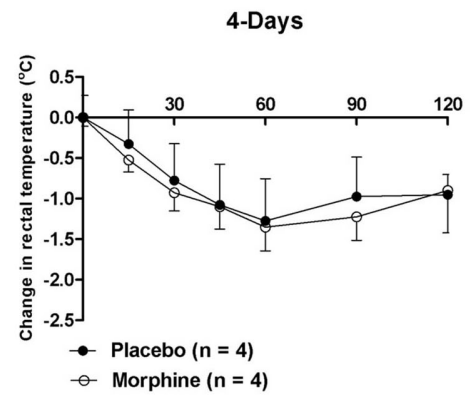

D

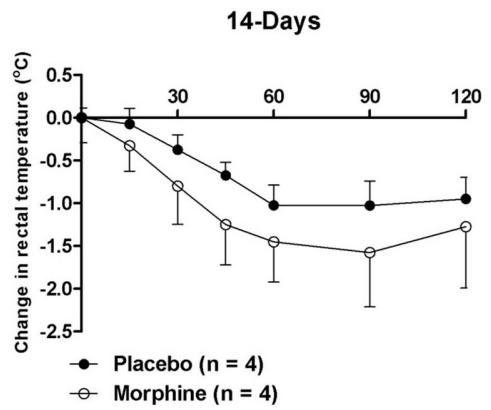

$\mathbf{E}$

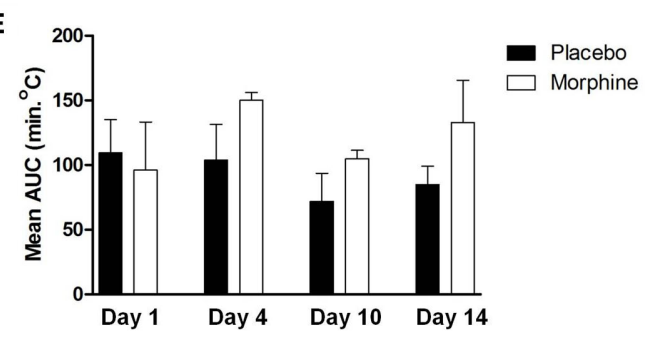

FIGURE 1 | (A-D) The change in body temperature produced by an acute challenge with morphine $(10 \mathrm{mg} / \mathrm{kg})$ in guinea pigs implanted with either placebo- or morphine-containing pellets at various time periods. Morphine produced a modest hypothermia that was not apparently altered by chronic treatment with morphine. (E) The calculated AUC values for the hypothermic effect of morphine in guinea pigs implanted with morphine pellets. The values were used to evaluate the total magnitude of the response to morphine $(10 \mathrm{mg} / \mathrm{kg})$. Ratios of AUC values were determined and are presented in Table 1. Statistically significantly different values are indicated by $(*)$. 
Table 1 | Influence of morphine exposure for various time periods after pellet implantation on the ratio of hypothermic and analgesic responses to morphine in vivo, maximum contractions to nicotine and inhibition of neurogenic twitches by DAMGO and 2-CADO of LM/MP preparations in vitro.

\begin{tabular}{|c|c|c|c|c|}
\hline & \\
\hline & 1 day & 4 days & 10 days & 14 days \\
\hline \multicolumn{5}{|c|}{ IN VIVO RESPONSES: RATIO OF MORPHINETO PLACEBOTREATED GROUP RESPONSE } \\
\hline Hypothermia AUC & $1.13(4)$ & $0.7(4)$ & $0.7(4)$ & $0.82(4)$ \\
\hline \multicolumn{5}{|c|}{ IN VITRO EXCITATORY RESPONSES: RATIO OF MORPHINE TO PLACEBOTREATED GROUP RESPONSE } \\
\hline Nicotine Maximum & $0.74(4)$ & $1.41 *(4)$ & $1.93^{*}(4)$ & $1.31(4)$ \\
\hline 2-CADO IC 50 values & $1.7(4)$ & $3.2 *(4)$ & $7.7 *(4)$ & $4.1^{*}(4)$ \\
\hline
\end{tabular}

Values depict the ratio of morphine to placebo treated group response.

The same animals or preparations obtained from those animals were used in each experiment so sample sizes are identical ( $n=4)$ for each response being evaluated. Values that are statistically significant $(p \leq 0.05)$ are indicated by $*$.

and morphine groups. As illustrated in Figures 2A-C, significantly lower mechanical pressure was required to elicit a paw withdrawal in the animals chronically exposed to morphine compared to the placebo at 1,4 , and 10 days suggesting that tolerance had developed in response to the chronic morphine exposure and persisted beyond a time when morphine plasma concentrations would be expected to be very low. A similar reduction in threshold pressure was observed in animals treated for 7 days (data not show). In animals treated for 14 (Figure 2E) as well as 17 and 21 days (data not shown), there was no reduction in mechanical pressure threshold suggesting that the tolerance that developed between days 1 and 10 had spontaneously declined by 14 days after pellet implantation. The magnitude of tolerance developed as determined by the AUC values was statistically significantly different at 1-10 days but not at 14 (Figure 2E; Table 1), 17, or 21 days (data not shown) post-implantation. These data suggest that the development of tolerance to the analgesic effects of morphine occurs over a time course that extends through at least 10 days and spontaneously recovers to normal levels between 10 and 14 days after pellet implantation and remains constant beyond that time.

\section{LM/MP ORGAN BATH ASSAY}

Chronic exposure to morphine resulted in the development of subsensitivity to both 2-chloroadenosine (CADO) and DAMGO at 4, 7, and 10 days following pellet implantation (Tables 1 and 2). As illustrated in Figure 3, concentration-response curves for the inhibitory effect of DAMGO depict a significant rightward shift at 4 and 10 days but not at 1 or 14 days after pellet implantation. The calculated geometric mean $\mathrm{IC}_{50}$ values of the morphine and placebo groups revealed a significant reduction in responsiveness to both DAMGO and 2-CADO (Tables 1 and 2). As indicated by the ratio of mean $\mathrm{IC}_{50}$ values presented in Table 2 , the reduction in responsiveness increased in magnitude from day 4 to day 7 after implantation and, for DAMGO, began to decline after day 10 to return to values that were not statistically significantly different by day 14. A similar reduction in responsiveness as shown by the rightward shift of the concentration-response curves, was observed with 2-CADO but the responsiveness remained reduced even at 14 days after pellet implantation. These data suggest that the development of reduced responsiveness (i.e., tolerance) assessed in this in vitro model system develops slowly and progressively, and spontaneously reverses back to normal levels. The calculated magnitude of rightward or loss of sensitivity shift (i.e., ratio of mean $\mathrm{IC}_{50}$ values) of the treated compared to the control group was nearly fourfold for DAMGO and threefold for CADO at 4 days and reached a maximum of seven- to eightfold for both agonists 7 and 10 days after treatment (Tables 1 and 2). In addition, the maximum inhibitory effect for each agonist (ranged from $85 \pm 5$ to $100 \%$ of the twitch amplitude) at each time period and was not statistically significantly different between the treatment groups as has been previously reported (Leedham et al., 1989).

The impact of chronic drug treatment on the contractile response of the LM/MP to nicotine (Table 2) revealed no statistically significant differences in the nicotine $\mathrm{EC}_{50}$ values following chronic morphine treatment at any time period tested. However, chronic morphine treatment did produce an elevation in the maximum response that was evident at 4 and 10 days but not at 1 and 14 days after pellet implantation (Figure 4). As indicated in Table 1, the maximum response to nicotine increased by 1.4 -fold at 4 days and was elevated nearly twofold at day 10 suggesting that the time period over which sensitivity to nicotine is increased lies between 4 and 14 days of treatment.

\section{DISCUSSION}

The goal of these studies was to determine if the change in responsiveness observed in vitro correlates with changes assessed in vivo following chronic exposure to morphine. The results clearly affirm the proposed hypothesis. However, a corollary hypothesis predicted the time course of tolerance expressed in the in vitro LM/MP preparation would be similar to that observed in an in vivo model system. The results indicate that tolerance to the analgesic effect of morphine can be defined by a time course of development and decline that is consistent with that observed in the LM/MP. A clear correlation between the activity in the guinea pig LM/MP 

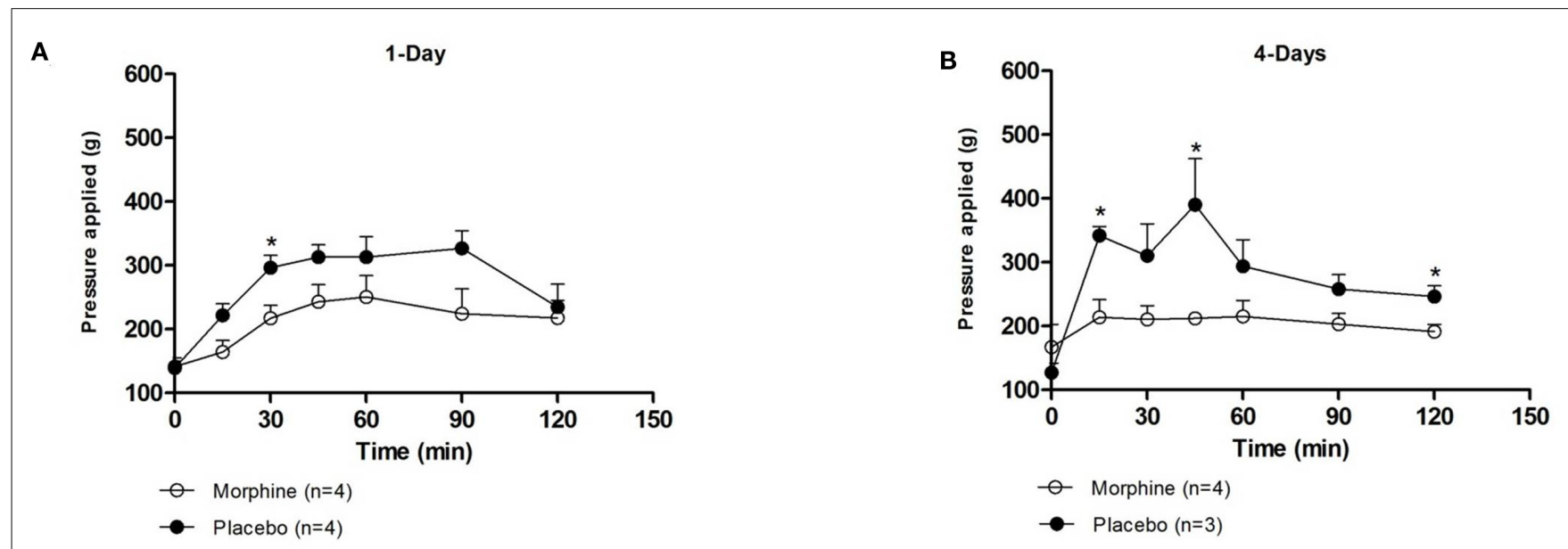

C
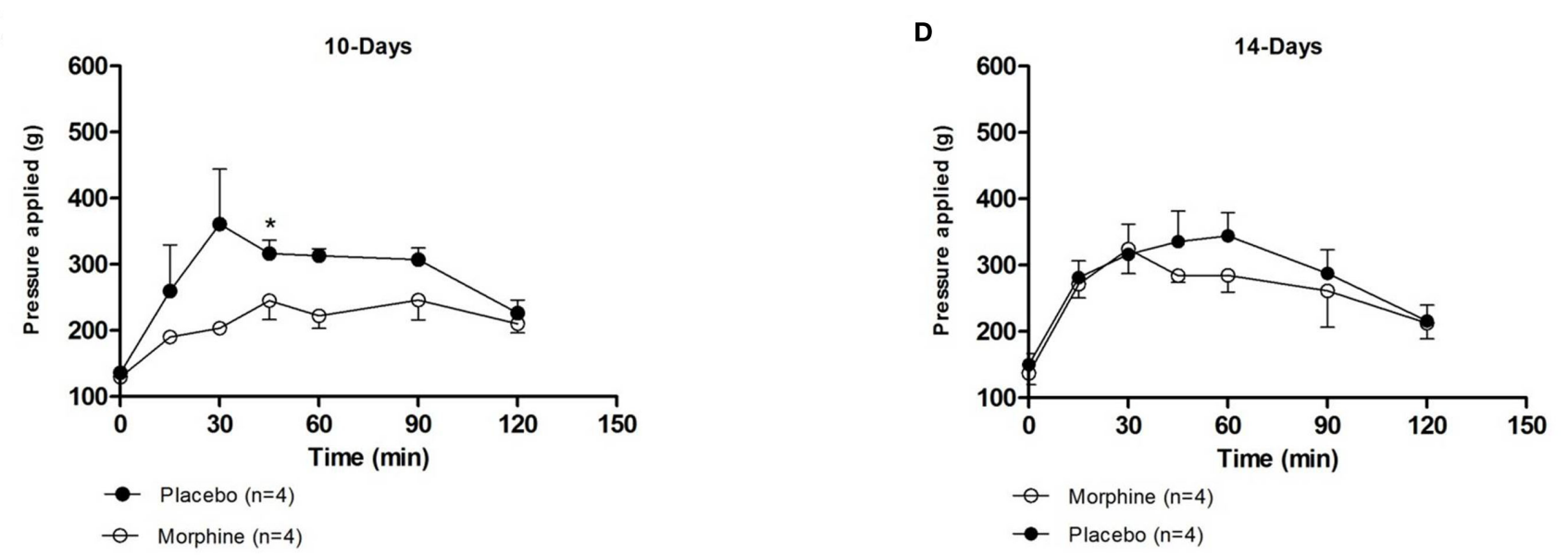

E

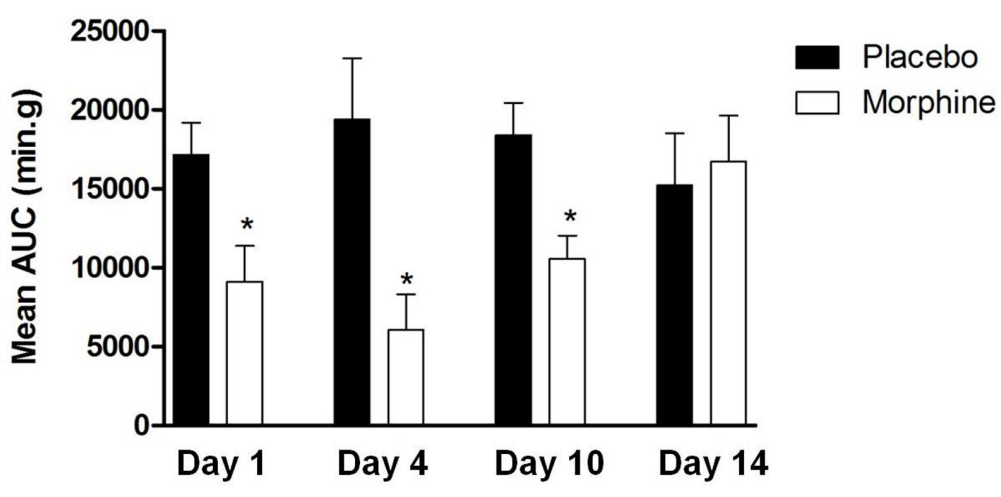

FIGURE 2 | (A-D) The analgesic effect produced by an acute morphine $(10 \mathrm{mg} / \mathrm{kg})$ administration in guinea pig implanted with either placebo- or morphine-containing pellets at various time periods. Analgesia was measured using the Randall-Sellito paw pressure test. (E) The calculated AUC values for

the analgesic effect of morphine in guinea pigs implanted with morphine pellets. The values were used to evaluate the total magnitude of the response to morphine $(10 \mathrm{mg} / \mathrm{kg})$. Ratios of AUC values were determined and are presented in Table 1. Statistically significantly different values are indicated by $(*)$. preparation model and analgesic activity of opioids in vivo has been previously documented (Kosterlitz and Waterfield, 1975). The development of reduced responsiveness to both 2-CADO and DAMGO, and the increased responsiveness to nicotine confirm earlier studies from several different laboratories demonstrating the heterologous nature of the change induced by chronic morphine treatment (Johnson et al., 1978; Taylor et al., 1988; Taylor and Fleming, 2001).

Recent work from this laboratory (Li et al., 2010) defined a similar time course for the development of functional heterologous 
Table 2 | Geometric Mean $\mathrm{EC}_{50}$ and $\mathrm{IC}_{50}$ values for agonist-induced responses of the LM/MP from animals implanted with morphine or placebo pellets for various times.

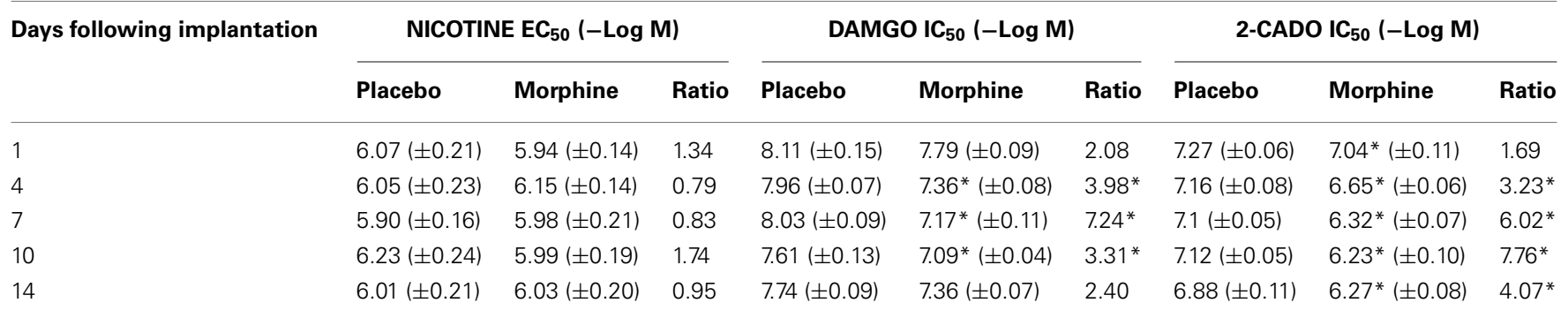

Sample sizes were 4 for each group at each time period.

Values that are significantly different from placebo $(p \leq 0.05)$ are indicated by $*$.

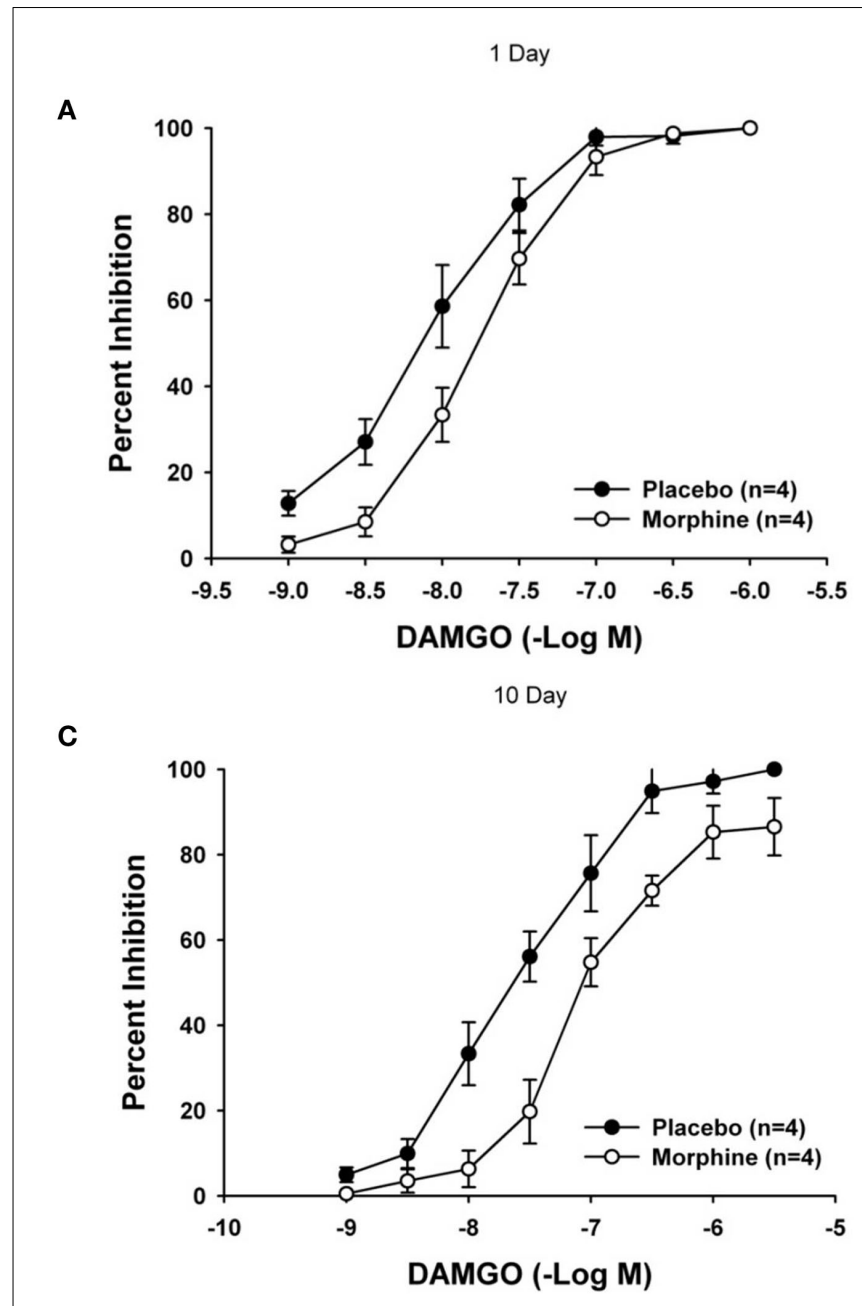

FIGURE 3 | Mean concentration-response curves illustrating the effect of chronic morphine treatment on DAMGO-mediated inhibition of neurogenic twitches in the LM/MP. Mean concentration-response curves in tissues obtained from animals 1 day
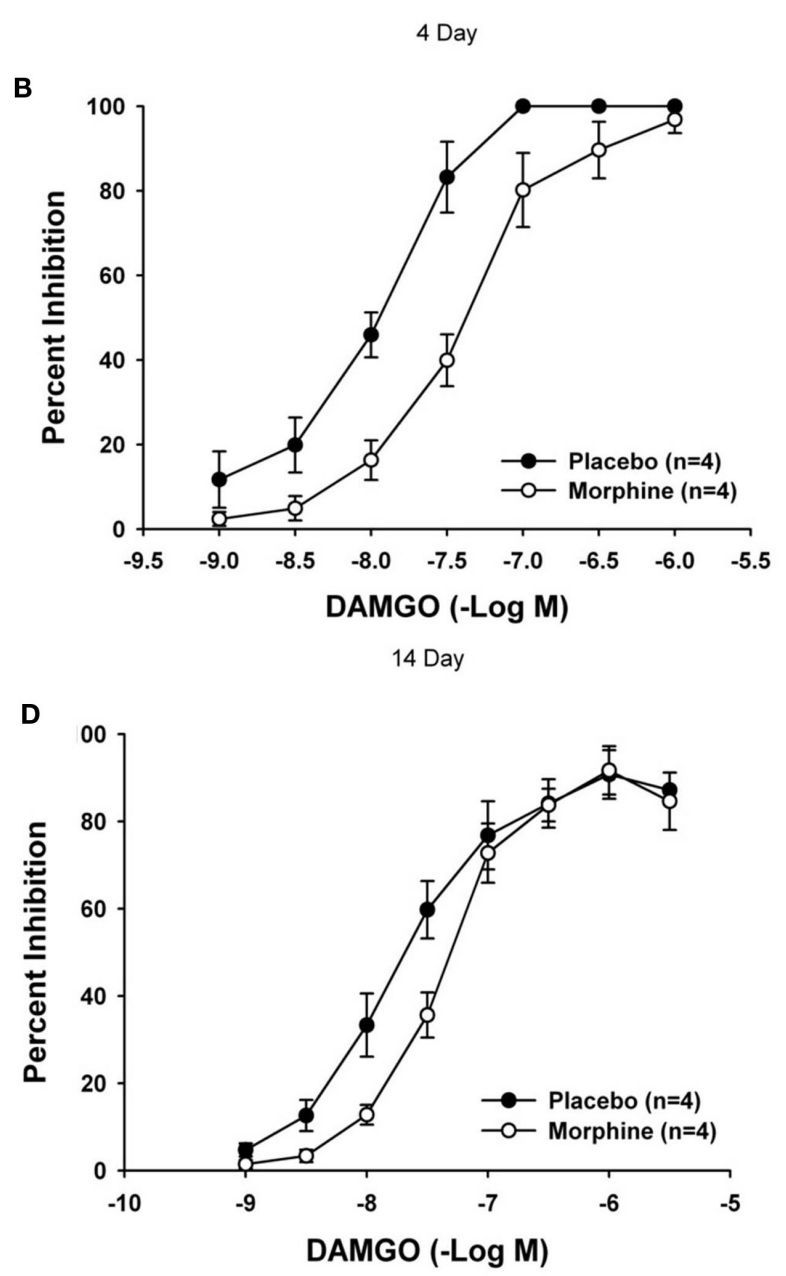

(A), 4-days (B), 10-days (C), and 14-days (D) after pellet implantation are shown. The magnitude of the degree of tolerance is assessed by comparing the geometric mean $I_{50}$ values (Table 2). A $p$ value of $\leq 0.05$ was considered statistically significant. tolerance in the guinea pig LM/MP following a single pellet implantation procedure. The fact that tolerance appears to develop over a time course that ranges between $>1$ and $<14$ days suggests an adaptive process induced by chronic morphine exposure that ultimately regulates cellular excitability. Though desensitization requires the presence of morphine, its maintenance does not. This 


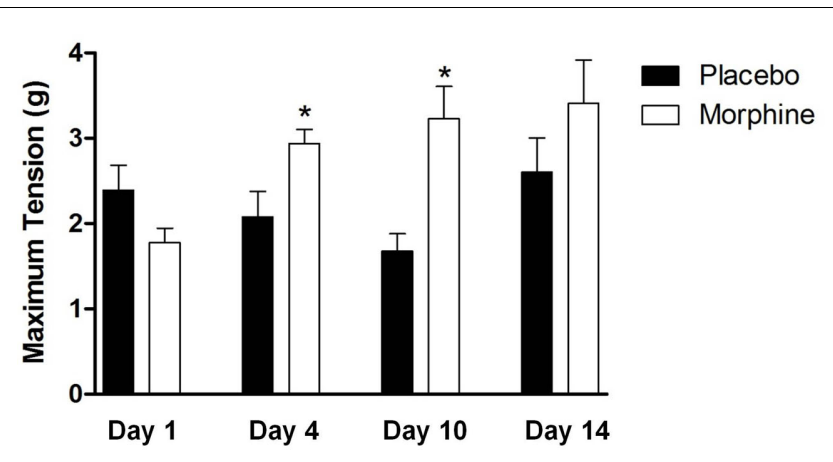

FIGURE 4 | The effect of chronic morphine treatment on the mean maximum responses of LM/MP preparations to nicotine. Statistically significant increases in the maximum response are identified by $*$. A $p$ value of $\leq 0.05$ was considered statistically significant.

notion is supported by the pharmacokinetics associated with morphine pellet implantation which shows peak morphine plasma concentrations by $4-6 \mathrm{~h}$ post-implantation that correlate to a "dumping" phase when the maximum antinociceptive effect is observed and gradually disappears over the next $36 \mathrm{~h}$ (Yoburn et al., 1985). In addition, almost $25 \%$ of the total morphine dose is absorbed within the first $24 \mathrm{~h}$. However, tolerance to the analgesic effects remained robust and heterologous tolerance began to develop in other response modalities by 4 days after implantation. This time period certainly extends beyond the $36 \mathrm{~h}$ time frame over which effective antinociceptive concentrations of morphine were observed in other studies (Yoburn et al., 1985). Furthermore, tolerance both in vivo and in vitro lasted beyond day 10 at which time morphine should be absent from the plasma.

The development of tolerance to the action of DAMGO and 2CADO on neurogenic twitches of the LM/MP has been reported in several different studies (Taylor et al., 1988; Leedham et al., 1989; Li et al., 2010). While most investigators use a 7-day treatment period, only a few have conducted extensive time course studies to characterize the functional changes in responsiveness (Leedham et al., 1989; Li et al., 2010). The data generated in this current study confirm the previous results and additionally demonstrates that the change in responsiveness to 2-CADO persisted well beyond the 14-day time period. This effect has not previously been reported though Li et al. (2010) did observe a significant reduction in responsiveness beyond 14 days that spontaneously resolved by 21 days. The data provided in this study differ from those described by Leedham et al. (1989) who found total reversal of tolerance at 14 days. Though no explanation is immediately apparent for the difference in these results, it is certainly possible that the development of tolerance beyond 14 days could well be a function of the sample size. However, since no change in responsiveness to DAMGO was observed and the enhanced response to nicotine diminished at that time, the persistent change in responsiveness to 2-CADO may be specific to that agonist since 2-CADO appears to produce a hyperpolarization of myenteric " $\mathrm{S}$ " neurons through a mechanism that is distinctly different from that of opioids (Meng et al., 1997). Thus, the continued reduction in responsiveness to 2-CADO may reflect the presence of complex adaptive changes in response to chronic morphine treatment (Taylor and Fleming, 2001). It is very likely that the adaptive processes employed by a cell, tissue, or animal may differ depending upon the nature of the agonist (McPherson et al., 2010). In spite of the apparent persistent change in responsiveness to 2CADO, the in vitro data appear to be consistent with previous studies and suggest that the LM/MP model may be very useful to evaluate the potential cellular mechanisms since it is predictive of the desensitization observed in vivo.

The lack of tolerance to the hypothermic effect of morphine is an intriguing observation that may be explained by one of several possibilities. First, the hypothermic effect produced by morphine is very modest in magnitude compared to that of other agents such as cannabinoids (Maguma and Taylor, 2011). The essential role of receptor activation that results in a robust physiological response and adaptation responsible for the development of tolerance is well documented (Taylor and Fleming, 2001) and has been further reinforced by previous studies showing that chronic use of sub-effective doses of opioids does not induce tolerance (Welch and Eads, 1999). The shallow hypothermic response observed with morphine may provide an insufficient stimulus to trigger changes in cell physiology that are expressed as heterologous tolerance in this model. Alternatively, the modest hypothermia observed upon morphine challenge may be due to the non-selective activation of mu- and kappa-opioid receptors which produce opposing effects (Rosow et al., 1980) with the former inducing hyperthermia the latter hypothermia (Adler and Geller, 1987; Spencer et al., 1988). Since the predominant effect observed was hypothermia, it is reasonable to assume that the response was primarily kappa receptor-mediated. The small but significant increase in mean body temperature at 4 and 7 days after pellet implantation could be an adaptation by the animal to unopposed small chronic hypothermia created by activation of kappa receptors. It is also possible that the change in body temperature could be due to altered responsiveness of the animal to the environmental temperature. Regardless of the mechanism, the change in mean body temperature is relatively minor $\left(0.7^{\circ} \mathrm{C}\right.$ at 4 days and $0.3^{\circ} \mathrm{C}$ at 7 days $)$.

This difference in receptor engagement has important implications to the current results and the comparison of data obtained in vivo versus data obtained in vitro. A similar circumstance of multiple receptors exists in the cholinergic myenteric neurons expressing both mu- and kappa-opioid receptors. Activation of either receptor population attenuates acetylcholine release and subsequent inhibition of muscle contractions (Leedham et al., 1991). However, the mechanism by which mu-opioid receptor activation inhibits acetylcholine release is by hyperpolarizing the " $\mathrm{S}$ " neurons while kappa receptor inhibit release by reducing the entry of calcium into the nerve terminals at the neuromuscular junction (Cherubini and North, 1985; Surprenant and North, 1985). This difference in the site and mechanism of action in response to mu- and kappa-opioid receptor activation may be responsible for the absence of tolerance to kappa-opioid receptor selective agonists in the LM/MP following chronic morphine treatment (Leedham et al., 1991). The possibility that the hypothermic effect in vivo may be due to kappa-opioid receptor activation further supports the correlation between data produced in the LM/MP with that observed 
in vivo. Hypothermia secondary to kappa-opioid receptor activation also suggests the potential existence of an adaptive change through membrane-potential mediated modification of cellular excitability.

The enhanced responsiveness to nicotinic receptor activation 7 days after morphine pellet implantation has been observed by a number of laboratories (Goldstein and Schulz, 1973; Johnson et al., 1978). However, no studies have evaluated the time course of the development of the change in responsiveness to determine whether the enhanced responsiveness shared a similar onset and decay as that observed for inhibitory agents (Leedham et al., 1989; Li et al., 2010). The observation that changes in cellular responsiveness to the excitatory effect of nicotine develop over a similar time course to the reduced responsiveness to both DAMGO and CADO in the LM/MP preparation further reinforces the idea that the adaptive change following chronic morphine treatment involves a modification in the membrane potential of myenteric "S" neurons (Meng et al., 1997). This enhanced sensitivity to excitatory agents and reduced responsiveness to inhibitory agents has been one of the foundations defining the heterologous nature of opioid tolerance (Goldstein and Schulz, 1973; Johnson et al., 1978). However, enhanced responsiveness develops to agents that depolarize myenteric " $S$ " neurons such as acetylcholine, serotonin, and nicotine and potassium but not to GABA and muscimol that produce contraction but do not depolarize "S" neurons (Goldstein and Schulz, 1973; Johnson et al., 1978; Leedham et al., 1992). The close correlation of the time course of reduced functional responsiveness to inhibitory agents and the reduction in the abundance of the $\alpha_{3}$ subunit of the ubiquitous

\section{REFERENCES}

Adler, M. W., and Geller, E. B. (1987). Hypothermia and poikilothermia induced by a kappa-agonist opioid and a neuroleptic. Eur. J. Pharmacol. 140, 233-237.

Biser, P. S., Thayne, K. A., Fleming, W. W., and Taylor, D. A. (2002). Na+, K+ ATPase alpha-subunit isoform distribution and abundance in guineapig longitudinal muscle/myenteric plexus after exposure to morphine. Brain Res. 931, 186-193.

Cherubini, E., and North, R. A. (1985). $\mathrm{Mu}$ and kappa opioids inhibit transmitter release by different mechanisms. Proc. Natl. Acad. Sci. U.S.A. 82, 1860-1863.

Connor, M., Osborne, P. B., and Christie, M. J. (2004). Mu-opioid receptor desensitization: is morphine different? Br. J. Pharmacol. 143, 685-696.

Goldstein, A., and Schulz, R. (1973). Morphine-tolerant longitudinal muscle strip from guinea-pig ileum. Br. J. Pharmacol. 48, 655-666.

Johnson, S. M., Westfall, D. P., Howard, S. A., and Fleming, W. W. (1978). Sensitivities of the isolated ileal longitudinal smooth muscle-myenteric plexus and hypogastric nerve-vas deferens of the guinea pig after chronic morphine pellet implantation. J. Pharmacol. Exp. Ther. 204, 54-66.

Kong, J. Q., Leedham, J. A., Taylor, D. A., and Fleming, W. W. (1997). Evidence that tolerance and dependence of guinea pig myenteric neurons to opioids is a function of altered electrogenic sodium-potassium pumping. J. Pharmacol. Exp. Ther. 280, 593-599.

Kosterlitz, H. W., and Waterfield, A. A. (1975). An analysis of the phenomenon of acute tolerance to morphine in the guinea-pig isolated ileum. Br. J. Pharmacol. 53, 131-138.

Leedham, J. A., Bennett, L. E., Taylor, D. A., and Fleming, W. W. (1991). Involvement of mu, delta and kappa receptors in morphine-induced tolerance in the guinea pig myenteric plexus. J. Pharmacol. Exp. Ther. 259, 295-301.

Leedham, J. A., Doak, N., Taylor, D. A., and Fleming, W. W. (1989). The development and reversal of the tolerance to morphine in the longitudinal smooth muscle-myenteric plexus preparation of the guinea pig. Life Sci. 45, 1483-1489.

$\mathrm{Na}^{+}, \mathrm{K}^{+}$-ATPase has been suggested to be the basis for the development of tolerance (Leedham et al., 1992; Kong et al., 1997; Biser et al., 2002; Li et al., 2010). It is tempting to speculate that these two distinct observations may be related and that the adaptive change in responsiveness defined by the altered excitability of myenteric neurons is related to the depolarization associated with the loss of electrogenic sodium pumping (Taylor and Fleming, 2001).

The results of these experiments indicate that the change in responsiveness to nicotine does develop and decay over a time course that is very similar to that seen with both other in vitro assessments of tolerance as well as in vivo evaluation of tolerance. The close correlation between the enhanced responsiveness to nicotine, the reduced response to multiple inhibitory agents seen in the current studies and the reduction in sodium pump alpha 3 subunit isoform provide a very strong circumstantial case for a functional relationship between these changes. The reduction in sodium pump abundance would lead to a membrane depolarization of excitable cells which would increase the responsiveness to excitatory agents like nicotine and serotonin and reduce responsiveness to inhibitors like DAMGO and 2-CADO. Furthermore, the data obtained support the hypothesis that functional assessment of the development of tolerance in vivo may share many common elements to that observed in vitro. In addition, the results of these studies support the idea that data assessing the mechanism(s) of development of tolerance obtained in the in vitro LM/MP preparation may be predictive of potential mechanisms that are responsible for the development of tolerance assessed using in vivo models.

Leedham, J. A., Kong, J. Q., Taylor, D. A., Johnson, S. M., and Fleming, W. W. (1992). Membrane potential in myenteric neurons associated with tolerance and dependence to morphine. J. Pharmacol. Exp. Ther. 263, 15-19.

Li, P., Maguma, H. T., Thayne, K., Davis, B., and Taylor, D. A. (2010). Correlation of the time course of development and decay of tolerance to morphine with alterations in sodium pump protein isoform abundance. Biochem. Pharmacol. 79, 1015-1024.

Maguma, H., and Taylor, D. A. (2011). The effect of chronic opioid vs. cannabinoid exposure on the expression of tolerance to morphine- or WIN-55, 212-2-induced analgesia and hypothermia in the guinea pig. Eur. J. Pharmacol. 660, 334-340.

McPherson, J., Rivero, G., Baptist, M., Llorente, J., Al-Sabah, S., Krasel, C., Dewey, W. L., Bailey, C. P., Rosethorne, E. M., Charlton, S. J., Henderson, G., and Kelly, E. (2010). $\mathrm{Mu}$-opioid receptors: correlation of agonist efficacy for signalling with ability to activate internalization. Mol. Pharmacol. 78, 756-766.

Meng, J., Malanga, C. J., Kong, J. Q., Taylor, D. A., and Fleming, W.
W. (1997). Hyperpolarizing effects of morphine, clonidine and 2chloroadenosine in myenteric neurons associated with tolerance to morphine. J. Pharmacol. Exp. Ther. 281, 41-47.

Randall, L. O., and Selitto, J. J. (1957). A method for measurement of analgesic activity on inflamed tissue. Arch. Int. Pharmacodyn. Ther. 111, 409-419.

Rosow, C. E., Miller, J. M., Pelikan, E. W., and Cochin, J. (1980). Opiates and thermoregulation in mice. I. Agonists. J. Pharmacol. Exp. Ther. 213, 273-283.

Schulz, R., and Goldstein, A. (1973). Morphine tolerance and supersensitivity to 5-hydroxytryptamine in the myenteric plexus of the guinea-pig. Nature 244, 168-170.

Spencer, R. L., Hruby, V. J., and Burks, T. F. (1988). Body temperature response profiles for selective $\mathrm{mu}$, delta and kappa opioid agonists in restrained and unrestrained rats. $J$. Pharmacol. Exp. Ther. 246, 92-101.

Surprenant, A., and North, R. A. (1985). mu-Opioid receptors and alpha 2adrenoceptors coexist on myenteric but not on submucous neurones. Neuroscience 16, 425-430. 
Taylor, D. A., and Fleming, W. W. (2001). Unifying perspectives of the mechanisms underlying the development of tolerance and physical dependence to opioids. J. Pharmacol. Exp. Ther. 297, 11-18.

Taylor, D. A., Leedham, J. A., Doak, N., and Fleming, W. W. (1988). Morphine tolerance and nonspecific subsensitivity of the longitudinal muscle myenteric plexus preparation of the guinea-pig to inhibitory agonists. Naunyn Schmiedebergs Arch. Pharmacol. 338, 553-559.
Welch, S. P., and Eads, M. (1999). Synergistic interactions of endogenous opioids and cannabinoid systems. Brain Res. 848, 183-190.

Yoburn, B. C., Chen, J., Huang, T., and Inturrisi, C. E. (1985). Pharmacokinetics and pharmacodynamics of subcutaneous morphine pellets in the rat. J. Pharmacol. Exp. Ther. 235, 282-286.

Conflict of Interest Statement: The authors declare that the research was conducted in the absence of any commercial or financial relationships that could be construed as a potential conflict of interest.

Received: 12 September 2011; accepted: 18 December 2011; published online: 10 January 2012.

Citation: Barrett DM, Maguma HT and Taylor DA (2012) Time course of altered sensitivity to inhibitory and excitatory agonist responses in the longitudinal muscle-myenteric plexus and analgesia in the guinea pig after chronic morphine treatment. Front. Pharmacol. 2:88. doi 10.3389/fphar.2011.00088

This article was submitted to Frontiers in Integrative and Regenerative Pharmacology, a specialty of Frontiers in Pharmacology.

Copyright (c) 2012 Barrett, Maguma and Taylor. This is an open-access article distributed under the terms of the Creative Commons Attribution Non Commercial License, which permits noncommercial use, distribution, and reproduction in other forums, provided the original authors and source are credited. 